\title{
CONDICIÓN SILVICULTURAL DE LA PALMERA Mauritia flexuosa L.f. EN EL ECOSISTEMA “AGUAJAL" DE PARINARI, LORETO, PERÚ
}

\author{
Luis FREITAS ${ }^{1}$, Hemilton FLORES ${ }^{2}$ \\ 1 Instituto de Investigaciones de la Amazonía Peruana (IIAP), Programa de Investigación en Manejo Integral del Bosque y \\ Servicios Ambientales (PROBOSQUES). Iquitos, Perú. E-mail: Ifreitas@iiap.org.pe \\ 2 Grupo de manejo Esperanza aguajales de Parinari, Distrito de Parinari, región Loreto
}

\section{RESUMEN}

El objetivo de este estudio es proveer información ecológica y silvicutural de Mauritia flexuosa para un adecuado manejo del ecosistema aguajal y de la especie en su medio natural. Se instaló una parcela permanente de muestreo de una hectárea, subdividida en 25 subparcelas de $20 \mathrm{~m}$ x $20 \mathrm{~m}$, y donde reportamos las existencias de Mauritia flexuosa distribuidas en cuatro estados de desarrollo: brinzales, latizales, fustales y palmeras maduras. La información levantada comprende parámetros de la estructura vertical y horizontal e información sobre la condición silvicultural de las palmeras: iluminación de la copa y forma de la copa. El bosque presenta 443 brinzales, 94 latizales, 45 fustales y 144 palmeras adultas. El 85.4\% de las palmeras adultas se desarrollan bajo condiciones óptimas de luz y el $73.6 \%$ poseen buenas formas de copa tipo círculo irregular. Más del 50\% de los fustales se desarrollan en condiciones deficientes de luz, y el $64.4 \%$ de plantas posee copas de forma de medio círculo. Existen bajas reservas de plantas en la categoría de latizales, sin embargo, el $75 \%$ de estas plantas se desarrollan en condiciones de luz aceptable. Las existencias de brinzales es alto, y el $47.9 \%$ de las plantas crecen en condiciones de luz deficiente, y el $52.1 \%$ en condiciones de luz aceptable.

PALABRAS CLAVE: Aguajales, Palmeras, humedales, ecología, Amazonía peruana.

\section{SILVICULTURAL CONDITION OF Mauritia flexuosa L.f. IN THE “AGUAJAL” ECOSYSTEM ZONE PARINARI, PERUVIAN AMAZON}

\begin{abstract}
The aim of this study was to provide ecological and silvicutural information of Mauritia flexuosa in order to improve forest management of swamp ecosystem and of the species in natural conditions. A permanent 1ha forest plot, divided in 25 subplots of $20 \mathrm{mx} 20 \mathrm{~m}$, was established, and all individuals of Mauritia flexuosa were classified in four life history stages: seedlings, saplings, juveniles and adults. Parameters of the vertical and horizontal structures and information on the silvicultural condition of palms were recorded: crown illumination and crown form. The forest had 443 seedlings, 94 saplings, 45 juveniles and 144 adult palms. The $85.4 \%$ of adult palms developed under optimum conditions of light, and $73.6 \%$ of palms have good cup form of an irregular circle. More than $50 \%$ of juveniles grow on poor light conditions, and $64.4 \%$ of juveniles have semi-circle crown shape. We found a low number of saplings, however, $75 \%$ of these plants grow on good light conditions. The number of seedlings is high, and $47.9 \%$ of these plants grow on poor light condition, and $52.1 \%$ in good light condition.
\end{abstract}

KEYWORDS: Aguajales, palms, swamp forests, ecology, Peruvian amazon. 


\section{INTRODUCCIÓN}

Los aguajales constituyen importantes ecosistemas amazónicos donde la palmera predominante es el aguaje (Mauritia flexuosa L.f), Encarnación $(1985,1993)$. Existen varios tipos de aguajales, los cuales son clasificados de acuerdo a la densidad de sus individuos. De esta forma tenemos aguajales densos o comunidades puras de Mauritia flexuosa, aguajales mixtos o asociaciones mixtas con renacos (Ficus sp y Coussapoa sp) y aguajales mixtos o comunidades dispersas de Mauritia flexuosa (IIAP-BIODAMAZ, 2005).

Estos ecosistemas juegan un rol importante desde el punto de vista social, ambiental y económico. El aguaje es una palmera nativa de la Amazonía que presenta un alto potencial de uso, especialmente los frutos, conocidos localmente como "aguajes" y que presentan altos contenidos de betacarotenos, vitamina $\mathrm{C}$ y E. (Vásquez et al., 20010; Del Castillo et al., 2005).

Se calcula que existen unos 6047058 de hectáreas de aguajales en la Amazonía peruana (OSINFOR, 2013), siendo la Reserva Nacional Pacaya Samiria, ubicada en la región Loreto, la zona de mayor concentración, en cuyo interior existen por lo menos un millón y medio de hectáreas de aguajales con diferentes densidades (CDC, 1993).

Muchas comunidades con poblaciones nativas, ribereñas o mestizas, asentadas a lo largo de los ríos de la Amazonía peruana dependen de la venta de frutos del "aguaje". Sin embargo, estos ecosistemas son aprovechados talando las palmeras a pesar de que la legislación prohíbe y regula esta forma de aprovechamiento. Se han realizado importantes estudios sobre los aguajales y el aguaje, principalmente sobre aspectos biofísicos, potencial alimenticio, propiedades bioquímicas de los frutos, caracterización genética, entomológicos, así como su comportamiento del cultivo en sistemas agroforestales (Delgado et al., 2007; Vásquez et al., 2008).

Los estudios sobre aspectos silviculturales para el manejo del ecosistema todavía son incipientes, se conoce que el aguaje es una especie heliófita (Cárdenas Valencia, 1985; Rivadeneyra, 1971), es decir, es una especie que requiere luz para su crecimiento y desarrollo. Así mismo, se cuenta con escasa información sobre su dinámica de regeneración, requerimientos ecológicos y silviculturales, así como la posición y comportamiento de los diferentes tamaños y estados de desarrollo de la vegetación en el bosque.

El presente trabajo tiene como objetivo aportar conocimientos relacionados a la estructura y las condiciones silviculturales, sus requerimientos de luz y forma de copas que presentan las palmeras de aguaje en diferentes estados de desarrollo, información que servirá para el manejo de la especie en su medio natural y para proveer criterios que faciliten la toma de decisiones técnicas para evitar la degradación de estos importantes ecosistemas.

\section{MATERIALES Y MÉTODOS}

El estudio se realizó en los "aguajales" pertenecientes a la comunidad de Parinari, situada en las coordenadas UTM 559818 E, 9488264 N, en la margen derecha del río Marañón, ámbito de la Reserva Nacional Pacaya Samiria, Distrito de Parinari, Provincia de Loreto, Región Loreto.

La vía principal de acceso a la zona es la fluvial, desde Iquitos aguas arriba por los ríos Amazonas y Marañón en un viaje de aproximadamente 24 horas en transporte público.

La temperatura media anual es de $26,03^{\circ} \mathrm{C}$, oscila a lo largo del año entre $25,21{ }^{\circ} \mathrm{C}$, media del mes de junio y julio, y $26,59{ }^{\circ} \mathrm{C}$, media del mes de noviembre; mientras que la temperatura media máxima anual es de $31,25^{\circ} \mathrm{C}$ y la temperatura media mínima anual $22,02^{\circ} \mathrm{C}$. La precipitación promedio anual es de $2.270 \mathrm{~mm}$, las precipitaciones mensuales varían entre $37 \mathrm{~mm}$ (febrero 2007) y $761 \mathrm{~mm}$ (julio 2002), (Baluarte, 2012).

Para el estudio de la vegetación se instaló una parcela permanente de muestreo (PPM) de $100 \mathrm{~m} \mathrm{x}$ $100 \mathrm{~m}$ (1 ha), la cual fue subdividida en 25 subparcelas de $20 \times 20 \mathrm{~m}$, realizándose un inventario de las existencias de Mauritia flexuosa (aguaje) distribuidas en cuatro estados de desarrollo o tamaño de las plantas: 1) brinzales, plantas de $0.3 \mathrm{~m}-1.5 \mathrm{~m}$ de altura; 2) latizales, plantas de $1.5 \mathrm{~m}-6 \mathrm{~m}$ de altura; 3) fustales, palmeras mayores que $6 \mathrm{~m}$ de altura con o sin estípite, que no hayan fructificado (juveniles) y 4) palmeras maduras, palmeras mayores que $6 \mathrm{~m}$ de altura con estípite y que hayan fructificado.

Todas las plantas fueron marcadas con una placa de aluminio numerada. La información levantada comprende parámetros de la estructura vertical y horizontal, así como información sobre la condición silvicultural de las palmeras (Iluminación de la copa y forma de la copa). Los parámetros evaluados, según estado de desarrollo de las plantas fueron los siguientes:

- Brinzales: Conteo de las plántulas, altura (m), iluminación de la copa.

- Latizales: $\mathrm{N}^{\circ}$ de planta, altura total (m), $\mathrm{N}^{\circ}$ de hojas, diámetro de la copa $(\mathrm{m})$, iluminación de la copa y forma de la copa. 
- Fustales: $\mathrm{N}^{\circ}$ de palmera, diámetro a la altura del pecho (Dap $\mathrm{cm})$, altura total $(\mathrm{m})$, altura del estípite $(\mathrm{m}), \mathrm{N}^{\circ}$ de hojas, diámetro de copa, iluminación de la copa y forma de la copa.

- Palmeras maduras: $\mathrm{N}^{\circ}$ de palmera, diámetro a la altura del pecho (Dap $\mathrm{cm})$, altura total $(\mathrm{m})$, altura del estípite $(\mathrm{m}), \mathrm{N}^{\circ}$ de hojas, sexo, estado fenológico, $\mathrm{N}^{\circ}$ de racimos, diámetro de copa, iluminación de la copa y forma de la copa.

La iluminación de la copa es una variable cualitativa que se refiere a la posición relativa de la copa de un árbol y sus vecinos de igual o mayor tamaño (Camacho 2000). Esta variable fue evaluada de acuerdo con la clasificación de Hutchinson (1993) (adaptada de Dawkins 1958) y tomando en cuenta las sugerencias de Pinelo (2000) y Louman et al (2001), de la siguiente forma: (1) Luz plena vertical y lateral (Buena), (2) Luz plena vertical (Buena), (3) Alguna luz vertical (Aceptable), (4) Luz lateral (Deficiente) y (5) Sin luz vertical ni lateral (Deficiente).

$\mathrm{Al}$ igual que la iluminación de la copa, la forma de la copa es una variable cualitativa que representa un índice de vigor del individuo. Se evaluó mediante la clasificación de Synnott (1991) (adaptada de Dawkins 1958) y las sugerencias de Pinelo (2000), teniendo en cuenta las tres dimensiones de la copa: (1) Círculo completo (Perfecta), (2) Círculo irregular (Buena), (3) Medio círculo (Tolerable), (4) Menos de medio círculo (Pobre) y (5) Solo una o pocas ramas (Muy pobre).

El lugar donde se instaló la parcela se localiza en las inmediaciones del caño Parinari, su ubicación en coordenadas UTM es 0556158 E, 9490119 N. El área estuvo sometida al aprovechamiento destructivo mediante la tala de aguaje y Euterpe precatoria (huasaí). A partir del año 1999, un grupo familiar (familia Flores Simón) de la comunidad de Parinari, tomó posesión parcial del área, aproximadamente 50 hectáreas de aguajal, denominándola con el nombre de "Esperanza". El área desde esa época está bajo manejo principalmente de la especie Mauritia flexuosa (aguaje), la técnica de manejo consiste en el escalamiento de la palmera para la cosecha de frutos, no se corta la palmera.

\section{RESULTADOS Y DISCUSIÓN}

\section{Parámetros más importantes del bosque}

El grupo de brinzales es el más numeroso, se reportaron 443 plántulas, mientras que los menos abundantes son los latizales con 94 individuos y fustales con 45 individuos. El grupo de palmeras maduras, mayores que $6 \mathrm{~m}$ de altura, están representados por 144 individuos, de los cuales, el $17.36 \%$ son plantas hembras y el $82.64 \%$ son machos.

La relación de sexos es variable, en aguajales con bajo aprovechamiento (menos de 5 aguajes cortados por hectárea) la relación es de $44 \%$ femeninos, $45 \%$ masculinos y $11 \%$ indeterminados (Freitas, 2014). Urrego (1987), en la Amazonía colombiana, reporta $49 \%$ de plantas femeninas y $51 \%$ de plantas masculinas.

La categoría de tamaño fustales (juveniles) presenta 45 individuos y representan el $23.81 \%$ del total de plantas (189 individuos) mayores que $6 \mathrm{~m}$ de altura. En este grupo (fustales), tres de ellas todavía no han desarrollado estípite, tienen una altura total promedio de $12.29 \mathrm{~m}$, variando entre 5 y $23 \mathrm{~m}$ (Tabla 1).

Tabla 1. Distribución de los parámetros más importantes del bosque según estados de desarrollo de las plantas de M. flexuosa L.f.

\begin{tabular}{lcccccc}
\hline $\begin{array}{c}\text { Categoría } \\
\text { de } \\
\text { regeneración }\end{array}$ & $\begin{array}{c}\text { Abundancia } \\
\mathbf{N} / \mathbf{h a}\end{array}$ & $\begin{array}{c}\text { Area basal } \\
\left(\mathbf{m}^{2} / \mathbf{h a}\right)\end{array}$ & $\begin{array}{c}\text { Dap } \\
\text { promedio } \\
(\mathbf{c m})\end{array}$ & $\begin{array}{c}\text { Altura Total } \\
\text { promedio } \\
(\mathbf{m})\end{array}$ & $\begin{array}{c}\text { Altura } \\
\text { promedio del } \\
\text { estípite }(\mathbf{m})\end{array}$ & $\begin{array}{c}\mathbf{N}^{\circ} \text { de } \\
\text { hojas }\end{array}$ \\
\hline Brinzales & 443 & - & - & 0.74 & - & 3 \\
\hline Latizales & 94 & - & - & 3.19 & - & 4 \\
Fustales & 45 & 2.4 & 26.69 & 12.29 & 8.99 & 6 \\
\hline Palmeras maduras & 144 & 12.05 & 32.19 & 23.26 & 18.67 & 10 \\
\hline TOTAL & 726 & 14.45 & - & - & - & - \\
\hline
\end{tabular}


De acuerdo al número actual de palmeras adultas y el alto porcentaje de aguajes masculinos, se puede inferir que este bosque fue sometido al aprovechamiento de sus frutos talando las palmeras. Inventarios forestales en aguajales reportan que la densidad varía mucho de un lugar a otro. De esta forma, en la Reserva Nacional Pacaya Samiria, se encontraron densidades de 230 a 240 aguajes adultos por hectárea (Mejía, 2000), 230 individuos de aguaje con estípite y con alturas alrededor de $10 \mathrm{~m}$ a más en una hectárea y muchos miles de plántulas menores a $1 \mathrm{~m}$ de altura (Khan et al., 1993).

En el piedemonte andino, al oeste de la Amazonía peruana, se encontró una densidad de 351 palmas con estípite y 297 acaulescentes juveniles por hectárea (Rivadeneyra, 1971). Otros estudios citados por Khan et al., (1993), presentan 246 palmas con estípite en una hectárea cerca de Iquitos en el río Itaya, 276 aguajes por hectárea en aguajal denso y 214 aguajes por hectárea en aguajal semidenso; mientras que Urrego (1987), en la región de Araracuara, río Caqueta, Colombia, registra la presencia de 272 plantas adultas por hectárea. Elárea basal del aguajal en estudio es de $14.45 \mathrm{~m}^{2}$, cantidad inferior a los reportados en aguajales no aprovechados, $18.21 \mathrm{~m}^{2}$ por hectárea que constituyen el $44.24 \%$ del área basal del bosque (Freitas, 1996).

La altura total promedio de las plantas dentro del grupo de palmeras maduras es de $23.26 \mathrm{~m}$ y una altura promedio del estípite de $18.67 \mathrm{~m}$. Para el caso de las plantas hembras maduras, que son las productivas, la altura promedio total y del estípite es de $21.84 \mathrm{~m}$ y $17.04 \mathrm{~m}$ respectivamente. El número total de plantas femeninas en la parcela es de 25 , de estas, 21 han fructificado (84\%) durante el presente año, de las cuales en las fechas que se hizo el estudio (setiembre, 2013) 3 palmeras habían sido cosechadas, las 4 restantes estaban en estado vegetativo.

De las 21 palmeras en fructificación, 12 de ellas (57\%) tienen más de 3 racimos y $9(43 \%)$ menos de 3 racimos; siendo el número promedio de racimos por planta de 2.8. Tomando en consideración los términos de referencia para la elaboración de planes de manejo forestal para palmeras (PRMRFFS, 2011), la cual establece que para el aprovechamiento se deben seleccionar individuos con tres o más racimos y alturas de fuste menor que $22 \mathrm{~m}$, en el área de estudio, todos los individuos femeninos pueden considerarse aprovechables de acuerdo a la altura del estípite, pero a nivel de número de racimos la cantidad de palmeras aprovechables será menor. Sin embargo, esta última aseveración es relativa, ya que probablemente la producción anual del número de racimos puede variar.

Para la aplicación de la norma mencionada, consideramos que deberían realizarse estudios más exhaustivos donde se analice principalmente las alturas totales de los individuos productivos con el fin de determinar si realmente son más abundantes en alturas menores que $22 \mathrm{~m}$. Por otro lado, con relación al número de racimos se debe tomar en consideración que estas pueden variar anualmente, ya que los problemas ambientales de constantes sequías e inundaciones pueden alterar la fenología y producción de las plantas.

\section{Iluminación de las copas}

La condición silvicultural de las palmeras, expresada por la cantidad de luz que reciben sus copas, muestra que el $54.86 \%$ de las plantas mayores que $6 \mathrm{~m}$ de altura del grupo de palmeras adultas, reciben plena iluminación superior, mientras que un $30.55 \%$ tiene las copas emergentes, es decir, reciben completa iluminación vertical y lateral (Tabla 2). En el caso de las plantas juveniles comprendidas en el grupo de fustales, de los 45 individuos, el $44.44 \%$ recibe alguna iluminación superior y el $51.11 \%$ iluminación lateral y solamente un $4.44 \%$ plena iluminación superior.

De forma general, para el grupo de palmeras maduras, podemos decir que el $85.41 \%$ de las plantas de esta categoría se encuentran bajo condiciones óptimas de luz, sin embargo, en el caso de los fustales (juveniles), considerada la población de plantas que reemplazará al grupo de palmeras adultas, más de la mitad de ellos se están desarrollando en condiciones deficientes de luz, de acuerdo a los tipos de iluminación establecidos por Pinelo (2000) y Louman et al (2001a).

En el caso de los brinzales, que son el grupo más abundante en el bosque, el $52.1 \%$ y $47.9 \%$ de las plantas reciben alguna iluminación superior (aceptable) e iluminación lateral (deficiente) correspondientemente, mientras que las plantas del grupo de latizales, la mayor cantidad de individuos, el 69\%, están recibiendo alguna iluminación superior (aceptable) (Tabla 2).

Existen bajas reservas de plantas en las categoría de latizales, 94 en la parcela de estudio. Sin embargo, casi tres cuartos del total de plantas se están desarrollando en condiciones de luz aceptable, pudiendo considerarse a este grupo como regeneración establecida, ya que las plantas han ganado un espacio de dominancia y tienen asegurada su permanencia en el bosque. 
Tabla 2. Condición silvicultural según iluminación de las copas para diferentes estados de desarrollo de plantas de M. flexuosa .L.f.

\begin{tabular}{|c|c|c|c|c|c|c|c|c|c|}
\hline \multirow[b]{2}{*}{ Parámetro } & \multicolumn{9}{|c|}{ Categorías de regeneración } \\
\hline & $\begin{array}{l}\text { Brin- } \\
\text { zales }\end{array}$ & $\%$ & $\begin{array}{l}\text { Lati- } \\
\text { zales }\end{array}$ & $\%$ & $\begin{array}{l}\text { Fus- } \\
\text { tales }\end{array}$ & $\%$ & $\begin{array}{l}\text { Palm. } \\
\text { Mad. }\end{array}$ & $\%$ & Total \\
\hline \multicolumn{10}{|l|}{ Iluminación copa } \\
\hline 1 emergente & - & - & - & - & - & - & 44 & 30.55 & 44 \\
\hline 2 Plena iluminación superior & - & - & - & - & 2 & 4.44 & 79 & 54.86 & 81 \\
\hline 3 Alguna iluminación superior & 231 & 52.1 & 69 & 73.4 & 20 & 44.44 & 18 & 12.5 & 338 \\
\hline 4 lluminación lateral & 212 & 47.9 & 25 & 26.6 & 23 & 51.11 & 3 & 2.08 & 263 \\
\hline 5 Ninguna iluminación directa & - & - & - & - & - & - & - & - & - \\
\hline TOTAL & 443 & 100 & 94 & 100 & 45 & 100 & 144 & 100 & 726 \\
\hline
\end{tabular}

En el grupo de brinzales, las 443 plantas reportadas en los levantamientos podrían estar indicando buenas condiciones de regeneración del bosque. Sin embargo, existe un alto porcentaje de plantas que se encuentran en condiciones de luz deficiente $(47.9 \%)$. En la actualidad no se cuenta con información sobre el comportamiento silvicultural de este grupo de plantas, es probable que el aguaje, por ser una especie de temperamento heliófito, necesite mejorar las condiciones de luz para mejorar su crecimiento.

De Paula Fernández (2002), evaluando la conservación del banco de plántulas en aguajales, ubicados en la zona del Acre-Brasil, encontró que al final de tres años un bajo porcentaje de plantas $(13 \%$ a $19 \%$ ) consiguieron sobrevivir, con poca variación en altura. Así mismo, sometió las plántulas de aguaje a diferentes intensidades de luz, las plántulas establecidas presentaron cierta sensibilidad al sombreamiento, el tratamiento a pleno sol presentó una mayor tasa de establecimiento (72\%), y el tratamiento a $70 \%$ de sombra una tasa menor $(46 \%)$, en sus desarrollo fueron tolerantes tanto al sol como a la sombra.

En el presente estudio, el grupo de brinzales se encuentran en lugares de poca luz y en sombra. Consideramos que tendrán mayores posibilidades de sobrevivir ya que se encuentran dispersas y no agrupadas como en un banco de plántulas.

\section{Forma de las copas}

Con relación a la forma de las copas, la mayor cantidad de palmeras consideradas en el grupo de adultas tienen la forma de copa círculo irregular, $61.37 \%$, y un $25.93 \%$ medio círculo, muy pocas tienen buena forma de copa, es decir, círculo entero, solamente un $9.5 \%$. En el caso del grupo de fustales, la forma de las copas difieren del grupo de palmeras maduras, ya que estas presentan un mayor porcentaje de plantas con formas de copas con medio círculo, $64.4 \%$, y el $22.2 \%$ copas de forma círculo irregular. El grupo de latizales presenta similares porcentajes de forma de copas que los fustales, donde predominan copas de medio círculo con $67 \%$ y un $33 \%$ círculo irregular (Tabla 3 ).

El análisis de los resultados nos permite mencionar que hay una relación directa entre la iluminación y forma de la copa, así, existe una correspondencia entre las plantas de categorías de tamaños menores (latizales y fustales) que presentan un alto porcentaje de copas con medio círculo (tolerables), las mismas que actualmente se encuentran en condiciones aceptables y deficientes de luz.

Similar razonamiento presenta Manta (1989), al comparar copas de baja calidad relacionándolas con clases diamétricas menores debido a la ausencia de luz, sobre todo cuando se trata de especies heliófitas.

La forma de las copas indican el vigor de las plantas, el alto porcentaje de plantas que presentan forma de copas circulo irregular y medio círculo podría estar influenciado por factores como competencia, plagas, enfermedades, pérdida de ramas o heridas (Louman, 2001b).

Se ha observado en el bosque en estudio, que existe una alta competencia entre las copas de los aguajes, así como con las copas de las especies latifoliadas en el dosel del bosque, una baja proporción de aguajes presentan las copas libres por encima del dosel. 
Tabla 3. Condición silvicultural según forma de las copas para diferentes estados de desarrollo de plantas de $M$. flexuosa L.f.

\begin{tabular}{lccccccc}
\hline \multirow{2}{*}{ Parámetro } & \multicolumn{7}{c}{ Categorías de regeneración } \\
\cline { 2 - 8 } & $\begin{array}{c}\text { Lati- } \\
\text { zales }\end{array}$ & $\%$ & $\begin{array}{c}\text { Fus- } \\
\text { tales }\end{array}$ & $\%$ & $\begin{array}{c}\text { Palm. } \\
\text { Mad. }\end{array}$ & $\%$ & Total \\
\hline Forma de copa & - & - & - & - & 18 & 12.50 & 18 \\
1: Círculo entero & 31 & 33 & 10 & 22.22 & 106 & 73.61 & 147 \\
2: Círculo irregular & 63 & 67 & 29 & 64.44 & 20 & 13.89 & 112 \\
3: Medio círculo & - & - & 6 & 13.33 & - & - & 6 \\
4: Menos que medio círculo & - & - & - & - & - & - & - \\
\hline 5: Solamente pocas hojas & 94 & 100 & 45 & 100 & 144 & 100 & 283 \\
\hline TOTAL & & & & & & & \\
\hline
\end{tabular}

\section{CONCLUSIONES Y RECOMENDACIONES}

El grupo de palmeras maduras se están desarrollando bajo condiciones óptimas de luz. Sin embargo, los fustales (juveniles) considerada la población de plantas que reemplazará a las palmeras maduras, más de la mitad de ellos se están desarrollando en condiciones deficientes de luz.

En el caso del grupo de latizales y fustales, las existencias de plantas son bajas, pero, el mayor número de individuos se están desarrollando bajo condiciones aceptables de luz y presentan altos porcentajes de plantas con copas de buena formación.

El bosque en estudio presenta serias restricciones para el manejo, debido a las bajas existencias de plantas del grupo de latizales y fustales y un alto número de brinzales con limitaciones de luz. Adicionalmente existe un bajo número de plantas femeninas que afectará la futura producción.

Realizar estudios de reclutamiento, mortalidad y crecimiento de plantas en diferentes estados de desarrollo, brinzales, latizales y fustales, en condiciones naturales y bajo diferentes tratamientos silviculturales en parcelas permanentes de muestreo.

\section{BIBLIOGRAFÍA CITADA}

Baluarte, J.R. 2012. Modelización del crecimiento de quince especies forestales comerciales del bosque aluvial inundable de la Amazonía Peruana. Tesis Doctoral, Universidad de Santiago de Compostela, Escola Politécnica Superior. Lugo, España. 231pp.

CDC. 1993. Evaluación ecológica de la Reserva Nacional Pacaya Samiria. Fase I. Informe presentado a la Fundación Peruana para la Conservación de la Naturaleza y The Nature
Conservancy, Employmenmt and Nature resources Sustainability Project. Centro de Datos para la Conservación. Lima, Perú. 156 pp.

Camacho C, M. 2000. Parcelas permanentes de muestreo en bosque natural tropical: guía para el establecimiento y medición. Turrialba, CR, CATIE: Serie Técnica. Manual Técnico no. 42. $52 \mathrm{pp}$.

Cárdenas Valencia, L. 1986. Estudio ecológico y diagnóstico silvicultural de un bosque de terraza media en la llanura aluvial del Río Nanay, Amazonía Peruana. Tesis Mag. Sc. Turrialba, C.R., Programa Universidad de Costa Rica/CATIE. 133 pp.

Del Castillo, D.; Otárola, E.; Freitas, L. 2006. La maravillosa palmera de la Amazonía Peruana. Instituto de Investigaciones de Investigaciones de la Amazonía Peruana. 51pp.

Delgado, C.; Couturier, G.; Mejía, K. 2007. Mauritia flexuosa (Areaceae: calamoideae), an Amazonian Palm with cultivation purposes in Perú. Fruits, 62 (3): 157- 169.

Delgado, C.; Vásquez, J.; Couturier, G.; Mejía, K., 2003. Relationship between Mauritia flexuosa and Eupalamides cyparissias in the Peruvian Amazon. Palms 47(2): 104-106

De Paula Fernandez, N.M. 2002. Estrategias de producción de sementes y establecimiento de plántulas de Mauritia flexuosa (Arecaceae) no vale do Acre, Brasil. Tese de Doutorado, Instituto Nacional de Pesquizas da Amazonia/Universidade Federal do amazonas, Manaus, Amazonas, Brasil. 203pp.

Encarnación, F. 1985. Introducción a la flora y vegetación de la Amazonía Peruana: Estado actual de los estudios, medio natural y ensayo de claves de determinación de las formaciones 
vegetales de la llanura Amazónica. Canollea 40(1):237-252.

Encarnación, F. 1993. El bosque y las formaciones vegetales en la llanura amazónica del Perú. Alma Mater 6: 95-114.

Freitas, L. 1996. Caracterización florística y estructural de cuatro comunidades boscosas de la llanura aluvial inundable en la zona de Jenaro Herrera, Amazonía Peruana. Instituto de Investigaciones de la Amazonía Peruana. 73pp. Doc. Tec. N²1. Iquitos, Perú.

Freitas, L. 2014. Impacto del aprovechamiento de Mauritia flexuosa L.f. sobre la estructura, producción y valor económico del ecosistema "aguajal" en la Amazonía Peruana. En revisión, Artículo enviado a la Revista Recursos Naturales y Medio Ambiente, CATIE, Turrialba-Costa Rica. 19pp.

González Rivadeneyra, M. 1971. Estudio sobre la densidad de poblaciones de aguaje (Mauritia sp) en Tingo María-Perú. Revista Forestal del Perú 5(1-2): 1-11.

Hutchinson, I. 1993. Puntos de partida y muestreo diagnóstico para la silvicultura de bosques naturales del trópico húmedo. Turrialba, CR, CATIE. 32pp. (Serie Técnica. Informe técnico no. 204. Colección Silvicultura y Manejo de Bosques Naturales no. 7).

IIAP-BIODAMAZ. 2004. Diversidad de vegetación de la Amazonía peruana expresada en un mosaico de imágenes de satélite. Documento técnico $\mathrm{N}^{\circ} 12$. Serie IIAP-BIODAMAZ, IquitosPerú. 68pp.

Kahn, F.; Mejía, K.; Moussa, F.; Gómez, D.; 1993. Mauritia flexuosa (Palmae), la más acuática de las palmeras amazónicas. 287-308. In Kahn, F.; Leon, B.; Young, K.R (compiladores). Las plantas vasculares en las aguas continentales del Perú. Instituto Francés de estudios andinos. Lima-Perú. 357pp.

Louman, B; Pinelo, G; Morales, J. 2001a. Informe de avances en el monitoreo de la dinámica del bosque en Petén, Guatemala. s.1., CONAP, CATIE, NPV. 27pp.

Louman, B.; Quiróz, D.; Nilsson, M. 2001b. Silvicultura de bosques latifoliados húmedos con énfasis en América Central. Turrialba, CR, CATIE. Serie Técnica. Manual Técnico no. 46. 265pp.
Manta, I. 1988. Análisis silvicultural de dos tipos de bosque húmedo de bajura en la vertiente atlántica de Costa Rica. Tesis de M. Sc. Centro Agronómico Tropical de Investigación y enseñanza. Turrialba, Costa Rica. 150pp.

Mejía, K. 2000. Palmerales de la Reserva Nacional Pacaya Samiria. Instituto de Investigaciones de la Amazonía Peruana. Informe final. 38pp.

Pinelo, M.G.I. 1997. Dinámica del bosque petenero: avances de investigación en Petén, Guatemala. Turrialba, CR, CATIE. 48pp. (Serie Técnica. Informe Técnico no. 296. Colección Manejo Forestal en la Reserva de la Biosfera Maya Petén, Guatemala no. 7).

Pinelo, M.G.I. 2000. Manual para el establecimiento de parcelas permanentes de muestreo en la Reserva de Biosfera Maya, Petén, Guatemala. Turrialba, CR, CATIE. 52pp. (Serie Técnica. Manual Técnico no. 40. Colección Manejo Forestal en la Reserva de la Biosfera Maya Petén, Guatemala no. 10).

PRMRFFS. 2011. Resolución ejecutiva directoral $N^{\circ}$ 62-2011. Programa Regional de Manejo de Recursos Forestales y de Fauna Silvestre. Iquitos, Perú. 3pp.

Synnott, TJ. 1991. Manual de procedimientos de parcelas permanentes para bosque húmedo tropical. Trad. J Valerio. CR, Instituto Tecnológico de Costa Rica. 103pp.

Urrego, L.E. 1987. Estudio preliminar de la fenología de la canangucha (Mauritia flexuosa L.f.). Colombia Amazonica, 2 (2): 57-81 .

Vásquez, P.; Freitas, L.; Sotero, V.; Paván, R.; Mancini-Filho, J. 2010. Chemical characterization and oxidative stability of the oils from three morphotypes of Mauritia flexuosa L.f. from the Peruvian Amazon. Grasas yAceites, 61(4): 390-397.

Recibido: 1 de setiembre del 2015

Aceptado para publicación: 15 de octubre del 2015 
